\title{
How to deal with revisions?
}

\author{
Goo-Hyun Mun
}

Associate Editor, Archives of Plastic Surgery

Department of Plastic Surgery, Samsung Medical Center, Sungkyunkwan University School of Medicine, Seoul, Korea

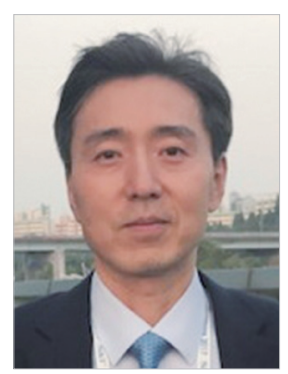

It is very uncommon for manuscripts submitted to peer-reviewed journals to be accepted directly, without a request for revision. Most submitted manuscripts go through a meticulous review process by several reviewers who are experts in the given subject; these reviewers may request major or minor revisions or even reject the manuscript. The revision of a manuscript is a crucial step in the publication process, particularly for medical research, as it can considerably improve the quality of the paper and its suitability for publication. Therefore, authors need to be well-prepared for handling returned manuscripts and dealing with comments from the editor and reviewers proficiently.

The editor's decision requesting the revision of a manuscript can be classified into two categories - minor and major revisions - although the specific terminology used may vary across journals $[1,2]$. When minor revisions are required, the reviewers have provisionally determined the manuscript to be appropriate for publication and have asked for only a few modifications, such as providing additional information or a deeper discussion, deleting redundant material, changing the style and/or format of the manuscript, and editing the language for clarity and grammaticality. These are generally not significant issues. Major revisions, in contrast, require a more fundamental reworking of the manuscript. The reasons for major revisions are diverse, including inconsistencies among different parts of the manuscript, an unclear presentation of the novelty of the findings, faulty deductions, a defective statistical analysis, irrelevant interpretations, and faulty relationships among the hypothesis, study design, and conclusions. Major revisions may require simply moving parts of the text around, or they may require the authors to reanalyze the data and rewrite the whole manuscript.
Such revisions necessitate conceptual reorganization, proper judgment, and the investment of considerable time and serious effort by the author. Herein, the author presents general considerations and several tips that can facilitate manuscript revision.

\section{GENERAL CONSIDERATIONS IN PREPARING THE REVISION}

An important point is that a request for either major or minor revision needs to be viewed positively [3], because it indicates that there is a chance that the manuscript can still be accepted and published, provided that all the queries and suggestions by reviewers are satisfactorily addressed. Upon receiving notice that major revisions of the manuscript will be required, authors may consider submitting it to other journals rather than revising it, as revision can be a very tiring and tricky process. In this context, however, it is recommended for most authors to resubmit their revised manuscript to the same journal, since the journal is likely to have expressed their interest in the authors' study by asking for revisions to be made.

When authors receive a decision letter requiring substantial revisions for the first time, they may think that the reviewers' comments are excessively negative and that their manuscript was not evaluated fairly. In this situation, it may be helpful to leave the comments for a couple of days rather than to react to the criticism immediately after receiving the reviews $[2,4]$. This short delay can allow time for the emotional response that may interfere with successful revision to subside.

After allowing a short delay, it is preferable for authors to start the revision promptly [4]. It is not recommended to wait until 
the last minute to complete the revision, particularly in cases of minor revision. If the re-submission is prompt, it is likely that the study will still be fresh in the minds of the reviewers, which can lead to a quick review and final acceptance. A long delay in re-submission, however, can give the editor and reviewers the potentially wrong impression that the study is not a priority for the authors or has serious problems that would be difficult to be fixed. This can reduce their enthusiasm and interest in the authors' work and may lead to adverse consequences. Of course, in cases of major revisions requiring extensive reorganization and re-writing, authors should carefully utilize the permitted time to ensure a comprehensive understanding of the reviewers' comments and to respond to them carefully, without being under pressure.

\section{TIPS FOR REVISING MANUSCRIPTS}

The " 3 golden rules" should be used when responding to reviewers' comments: answer completely, answer politely, and answer with evidence [5].

\section{Answer completely}

Authors have the responsibility to respond to all the queries raised by the editor and reviewers, either minor or major ones, when revising a manuscript. That is, no point of discussion should be left unexplained or unanswered, and the revision should be conducted in an itemized fashion with point-by-point responses to all the reviewers' comments $[2,5]$.

Moreover, authors should clearly state which parts are revised and include changes to the text of the manuscript in the letter to the editor, rather than just addressing the reviewers' comments. This prevents unnecessary confusion for the editor and reviewers while they conduct another review of the revised manuscript. It allows them to save the time and effort they would have spent seeking out where the modifications were made in the revised document.

\section{Answer politely}

Needless to say, while preparing the revision, it is not recommended for authors to criticize reviewers' comments or to enter into an argument with them [5]. Authors should keep in mind that the revised manuscript will be sent back to the same reviewers for another review, so it is preferable for all communications related the revision to be polite and written in a dispassionate manner. Additionally, authors should think of editors and reviewers as collaborators who have made efforts to improve the authors' work, not as adversaries.

\section{Answer with evidence}

All comments and responses should be stated based on evidence, with citations of the relevant existing literature or additional analyses. This is particularly important in situations where the authors disagree with the reviewers' comments [5]. Although it is a general rule in revising manuscripts that the editor and reviewers are right, disagreements can develop with regard to some of their comments. When authors feel that certain comments are inaccurate or stem from a misunderstanding of their work, they can rebut those points. However, in that situation, authors should provide a coherent argument supported by evidence, in which they clearly state the reasons for disagreement, rather than just saying "we disagree."

\section{Diligent refinement of the manuscript before initial submission}

In addition to those traditional golden rules, one of the cleverest strategies for revising a manuscript is to prevent difficult revisions by anticipating reviewers' comments before the initial submission of the manuscript to the journal. A thorough exploration of the potential weak points of a study, and addressing them as much as possible before the initial submission, may draw amicable responses from the editor and reviewers, potentially allowing tricky revisions to be avoided and making the publication process easy.

\section{SUMMARY}

Revising manuscripts is often challenging and may be a task that authors do not want to face, but it is a crucial step for significantly improving authors' work before the final publication. Understanding these general rules and the several tips discussed above may be helpful for authors in dealing with comments from editors and reviewers.

\section{NOTES}

\section{Conflict of interest}

No potential conflict of interest relevant to this article was reported.

\section{REFERENCES}

1. Dogra S, Sarangal R. Tits and tots of revising a manuscript. Indian J Dermatol Venereol Leprol 2013;79:733-6.

2. Provenzale JM. Revising a manuscript: ten principles to guide success for publication. AJR Am J Roentgenol 2010; 195:W382-7. 
3. Peh WC, Ng KH. Dealing with returned manuscripts. Singapore Med J 2009;50:1050-2.

4. Bhargava P, Agrawal G. From the editor's desk: a systematic guide to revising a manuscript. Radiol Case Rep 2015;8:824.

5. Williams HC. How to reply to referees' comments when submitting manuscripts for publication. J Am Acad Dermatol 2004;51:79-83.
Correspondence: Goo-Hyun Mun

Department of Plastic Surgery, Samsung Medical Center, Sungkyunkwan University School of Medicine, 81 Irwon-ro, Gangnam-gu, Seoul 06351, Korea

Tel: +82-2-3410-2233, Fax: +82-2-3410-0036, E-mail: supramicro@gmail.com

This article was presented at the 9th Congress of the World Society of Reconstructive Microsurgery on June 14-17, 2017.

Received: 5 Dec 2017 • Revised: 26 Jan 2018 • Accepted: 27 Jan 2018

pISSN: 2234-6163・ elSSN: 2234-6171

https://doi.org/10.5999/aps.2017.01816 • Arch Plast Surg 2018;45:99-101 